\title{
Profile of salivary gland flow dysfunctions in patients with differentiated thyroid carcinoma submitted to radioiodine therapy
}

\author{
Anna Clara Fontes Vieira', Aline Sampaio Lima Rodrigues ${ }^{1}$, \\ Maria Carmen Fontoura Nogueira da Cruz ${ }^{1,2}$, Fernanda Ferreira Lopes ${ }^{1,2}$
}

\author{
'Department of Dentistry, Dental School, Federal University of Maranhão, São Luís, MA, Brazil \\ ${ }^{2}$ Oral Pathology Department, Dental School, Federal University of Maranhão, São Luís, MA, Brazil
}

\begin{abstract}
Aim: To evaluate the effects of radioiodine therapy on salivary flow in patients with differentiated thyroid cancer. Methods: A sample comprising 88 patients submitted to ablation with iodine 131 was included in the study. The patients were submitted to sialometry and evaluation of the presence of xerostomia before, 10 days and 3 months after radioiodine therapy. Results: Xerostomia was observed in $36.4 \%$ of the patients before radioiodine therapy, $59.15 \%$ at 10 days after therapy, and $25 \%$ at 3 months after therapy. Significant differences were observed in non-stimulated salivary flow rates between the second and third evaluations $(p \leq 0.020)$ and in stimulated salivary flow between the first and second evaluations $(p \leq 0.010)$. Conclusions: The results suggest that changes in salivary flow resulting from radioiodine therapy are more pronounced during the first weeks after treatment and seem to regress after 3 months.
\end{abstract}

Keywords: thyroid gland tumors, iodine radioisotopes, salivary glands, saliva, xerostomia, hyposalivation.

\section{Introduction}

Differentiated thyroid carcinoma is a curable tumor, especially when diagnosed early. The most adequate treatment istotal thyroidectomy followed by actinic ablation with radioactive iodine (iodine 131) and has a very good prognosis (in more than $80 \%$ of cases) with excellent long-term survival, similar to that of the population that never had cancer ${ }^{1}$.

Iodine 131 is taken up by the thyroid through the sodium/iodide symporter (NIS), a protein that is also expressed in other tissues such as the salivary glands, stomach and breast. As a consequence, iodine 131 can cause damage to these structures since it is absorbed at these sites after administration of therapeutic $\operatorname{doses}^{2-4}$. The beta radiation of iodine 131 exerts cytotoxic effects on the salivary glands because these organs are highly radiosensitive ${ }^{5}$. All salivary glands are involved in the transport of radioactive iodine into the saliva ${ }^{6}$.

Saliva is essential to maintain adequate oral functions such as lubrication, chewing and swallowing, speech, oral $\mathrm{pH}$ balance, taste perception, and cleanliness. Quantitative and qualitative changes in salivary flow can compromise these functions. Consequently, subjects with salivary gland dysfunction are more susceptible to periodontal disease, rampant caries, and fungal and bacterial oral 
infections ${ }^{7}$. Longitudinal studies investigating the progression and intensity of salivary gland dysfunction in patients submitted to ablation with iodine 131 are scarce in the literature ${ }^{2,4,8-9}$.

The objective of the present study was to evaluate the effects of iodine 131 on the salivary glands by subjective symptom of dryness in the mouth (xerostomia) and reduced salivary flow (hyposalivation) of patients with differentiated thyroid carcinoma submitted to radioiodine therapy. Xerostomia and salivary flow were compared before and after therapy to establish the progression and frequency of salivary changes considering the high survival rate of these patients.

\section{Material and methods}

A clinical longitudinal study with of 88 patients with differentiated thyroid carcinoma (papillary and follicular) who underwent complementary treatment with high single doses of iodine 131 (100 to $150 \mathrm{mCi}$ ). The patients were treated at the Radioiodine Therapy Service of Instituto Maranhense de Oncologia Aldenora Bello and had not used drugs to treat other comorbidities.

The collected data were: personal identification (name, age, gender, educational level and marital status), anatomopathological definition of the tumor, data of radioiodine therapy (dose), presence or absence of xerostomia and salivary flow. Xerostomia and salivary flow were evaluated at three time points: before radioiodine therapy, 10 days after treatment (when a complete body scanning for the detection of metastases was done), and after 3 months. Xerostomia was evaluated by subjective symptoms of dryness in the mouth during anamnesis, and classified as present or absent. The patients did not receive any recommendation for home salivary gland stimulation, during the period of this study. Stimulated and non-stimulated whole saliva samples were collected for analysis of salivary flow ${ }^{10}$. The patients did not drink or eat 90 min before sialometry. All tests were performed at the same time $(13: 00$ to $15: 00 \mathrm{~h})$ to minimize variations in salivary secretion ${ }^{11}$. Descriptive statistics was used for analysis and the results were compared by the paired Student's t-test, adopting a 5\% significance level.

The study was approved by the Ethics Committee of the Federal University of Maranhão (protocol 23115005754/ 2009-00) and all patients signed an informed consent form.

\section{Results}

In a convenience sample of 88 patients submitted to ablation with iodine 131, differentiated thyroid carcinoma was more prevalent among women $(n=80)$ than men $(n=8)$. Patient age ranged from 18 to 72 years (mean: 43.4 years). Seventy-seven patients had papillary carcinoma and 11 had follicular carcinoma. Most participants $(52.3 \%)$ were married, $5.7 \%$ were illiterate, and $39.8 \%$ completed high school.

Saliva was collected initially from 88 patients, but only 25 continued to participate in the study for sialometry 3 months after the administration of iodine 131. A decrease in the mean non-stimulated and stimulated salivary flow rates was observed between the first (before radioiodine therapy) and second evaluation (10 days after therapy). In contrast, mean non-stimulated and stimulated salivary flow increased between the second and third evaluation (3 months after therapy). These differences were statistically significant for non-stimulated salivary flow between the second and third evaluations $(p \leq 0.020)$ and for stimulated salivary flow between the first and second evaluations $(\mathrm{p} \leq 0.010)$ (Tables 1 and 2$)$.

Xerostomia before radioiodine was reported by $36.4 \%$ of the patients. After 10 days of radioiodine therapy, 71 (100\%) participants were reassessed and 42 (59.15\%) reported xerostomia. Twenty patients did not report xerostomia prior to therapy. After 3 months, only $5(25 \%)$ among the 25 $(100 \%)$ patients had xerostomia, and three of these five reported symptoms prior to therapy (Table 3).

\section{Discussion}

It is important to provide best-quality care to patient with differentiated thyroid carcinoma, so knowledge of

Table 1. Comparison of non-stimulated salivary flow before radioiodine therapy (1st evaluation), 10 days (2nd evaluation) and 3 months (3rd evaluation) after therapy in 25 patients submitted to thyroidectomy*.

\begin{tabular}{llllll}
\multicolumn{5}{c}{ Non-stimulated salivary flow } \\
1st evaluation & \multicolumn{5}{c}{ 2nd evaluation } \\
Mean & SD & Mean & SD & $T$ & $p$ \\
0.4384 & 0.3039 & 0.3600 & 0.2540 & 1.383 & 0.179 \\
\hline 1st evaluation & & 3rd evaluation & & & \\
Mean & SD & Mean & SD & $T$ & $p$ \\
0.4384 & 0.3039 & 0.5428 & 0.4564 & -1.074 & 0.293 \\
\hline 2nd evaluation & & 3rd evaluation & & & \\
Mean & SD & Mean & SD & $T$ & $p$ \\
0.3600 & O.2540 & 0.5428 & 0.4564 & -2.491 & 0.020 \\
\hline
\end{tabular}

Results are reported as mean and standard deviation (SD). $p<0.05$ (paired $t$-test).

*Only 25 participants were submitted to sialometry 3 months after the administration of iodine 131 . 
Table 2. Comparison of stimulated salivary flow before radioiodine therapy (1st evaluation), 10 days ( 2 nd evaluation) and 3 months (3rd evaluation) after therapy in 25 patients submitted to thyroidectomy*.

\begin{tabular}{llllll}
\hline \multicolumn{5}{c}{ Stimulated salivary flow } \\
1st evaluation & \multicolumn{5}{c}{ 2nd evaluation } \\
Mean & SD & Mean & SD & $t$ & $p$ \\
1.5808 & 0.877 & 1.4144 & 0.9452 & 2.780 & 0.010 \\
\hline 1st evaluation & \multicolumn{5}{c}{ 3rd evaluation } \\
Mean & SD & Mean & SD & $t$ & $p$ \\
1.5808 & 0.877 & 1.6864 & 1.0774 & -0.815 & 0.423 \\
\hline 2nd evaluation & \multicolumn{5}{c}{ 3rd evaluation } \\
Mean & SD & Mean & SD & $t$ & $p$ \\
1.4144 & 0.9452 & 1.6864 & 1.0774 & -1.992 & 0.058 \\
\hline
\end{tabular}

Results are reported as mean and standard deviation (SD). $p<0.05$ (paired $t$-test). *Only 25 participants were submitted to sialometry 3 months after the administration of iodine 131.

Table 3. Distribution of patients with xerostomia before, after 10 days and after 3 months of radioiodine 131 therapy.

\begin{tabular}{llll}
\hline Xerostomia & Before $^{*}$ & ${\text { After } 10 \text { days }^{*}}^{*}$ & After 3 months \\
& $n=88(100 \%)$ & $n=71(100 \%)$ & $n=25(100 \%)$ \\
Present & $32(36.4 \%)$ & $42(59.15 \%)$ & $5(25 \%)$ \\
Absent & $56(63.6 \%)$ & $29(40.85 \%)$ & $20(75 \%)$ \\
\hline
\end{tabular}

*Radioiodine 131 therapy

complications and associated factors to high doses of radioactive iodine therapy $\left(\geq 100 \mathrm{mCi}\right.$ ) must be investigated ${ }^{12}$. There are few Brazilian studies about repercussion of iodine ablation on the salivary glands ${ }^{12-13}$. Although xerostomia was evaluated in these studies, no measurement of salivary flow to diagnose hyposalivation was done in these investigations.

The present study showed an increase of patients with symptoms of xerostomia from $36.4 \%$ (before radioiodine therapy) to $59.15 \%$ (10 days after therapy). After 3 months, only $5(25 \%)$ of the 25 reevaluated patients reported this symptom. Similar results were described in a longitudinal study in which the population was submitted to more than one evaluation and a decrease in the percentage of patients with xerostomia was observed several months after treatment ${ }^{12}$. That study had a follow-up period of 3 months while in the preent investigation the follow up period was 8 months ${ }^{12}$.

Caglar et al. ${ }^{14}$ evaluated subjective symptoms of salivary gland dysfunction in 39 patients submitted to radioiodine therapy. Twenty-one (54\%) patients developed xerostomia after ablation with iodine 131. In contrast, Grewel et al. ${ }^{9}$ evaluated retrospectively 262 patients in the first year of follow-up after treatment with high doses of iodine 131 and reported $17 \%$ of xerostomia. This incidence of xerostomia is similar to the $25 \%$ observed in the present study after 3 months of radioiodine therapy. However, in both cited studies salivary flow was not measured as in the present investigation.

Both non-stimulated and stimulated mean salivary flow rates decreased between the first (before radioiodine therapy) and second (10 days after therapy) evaluation. In contrast, an increase of non-stimulated and stimulated mean salivary flow rates was observed between the second and third (3 months after therapy) evaluations, i.e., salivary flow rates tended to return to baseline after 3 months. These findings suggest transient alterations in salivary flow as demonstrated by the observation of significant differences in non-stimulated salivary flow between the second and third evaluations $(\mathrm{p} \leq 0.020)$ and in stimulated salivary flow between the first and second evaluations $(\mathrm{p} \leq 0.010)$. Although there are few data about salivary gland dysfunction after radioiodine therapy, apparently there is higher prevalence of transient alterations ${ }^{9}$.

There are various longitudinal studies about postablation salivary gland dysfunction, but they are based on subjective prospective analyses (interviews and questionnaires) of glandular function ${ }^{8-9,12}$. Generally, objective analysis of glandular function has been done by scintigraphy, but most of these studies applied a cross-sectional method to demonstrate the occurrence of salivary disorders within months after treatment ${ }^{13-14}$. Prospectively, Solans et al. ${ }^{3}$ evaluated glandular dysfunction by scintigraphy over a period of 3 years. However, the exam was performed annually, i.e., the first evaluation of salivary gland dysfunction was made only one year after treatment. Contrastingly, in the present study the glandular function was evaluated objectively in 25 patients at three different time points (before, 10 days and 3 months after radioiodine therapy). Analysis by sialometry showed reduced salivary flow as early as 10 days after treatment. This finding indicates the need for complete dental follow-up of these patients since the time of admission for iodine 131 treatment.

There is no consensus on how to prevent adverse effects of ablation with high doses of radioactive iodine ${ }^{12}$. Several protocols for the prevention of salivary gland injury are used. The most common recommendation is sucking lemon candies or ingestion of lemon juice during the period of iodine 131 treatment $^{15}$.

Considering the definition of non-stimulated and stimulated hyposalivation (salivary flow rate $<0.1 \mathrm{~mL} / \mathrm{min}$ and $<0.7 \mathrm{~mL} / \mathrm{min}$, respectively) ${ }^{14}, 7$ of the studied patients had non-stimulated hyposalivation and 18 patients had stimulated hyposalivation at baseline. The prevention of salivary gland injuries after radioiodine ablation is of utmost importance because salivary gland dysfunction as an adverse effect of this therapy may turn the patients more susceptible to carious lesions and periodontal problems ${ }^{16}$.

The results of this study suggest that changes in salivary flow resulting from radioiodine therapy are more pronounced during the first weeks after treatment and it seems to retreat after 3 months. However, further clinical studies are needed for evaluating qualitative changes of salivary composition in patients undergoing radioiodine therapy. After radioiodine therapy, atients require efficient measures to prevent or reduce salivary gland dysfunction in order to improve their quality of life.

\section{Acknowledgements}

We thank Capes/ Education and Culture Government for the Master's fellowship granted to Anna Clara Fontes Vieira. We also thank FAPEMA for publishing support and 
Dr. Ibrahim Assub and the Nuclear Medicine Clinic of Maranhão for providing the physical space for sample collection and for the interview with the volunteers of the study.

\section{References}

1. Ward LS, Assumpção LV. Thyroid cancer: prognostic factors and treatment. Arq Bras Endocrinol Metabol. 2004; 48: 126-36.

2. Van Nostrand D. Sialoadenitis secondary to 131I therapy for well-differentiated thyroid cancer. Oral Dis. 2011; 17: 154-61.

3. Solans R, Bosch JA, Galofré P, Porta F, Roselló J, Selva-O'Callagan A, et al. Salivary and lacrimal gland dysfunction (sicca syndrome) after radioiodine therapy. J Nucl Med. 2001; 42: 738-43.

4. Almeida JP, Sanabria AE, Lima EN, Kowalski LP. Late side effects of radioactive iodine on salivary gland function in patients with thyroid cancer. Head Neck. 2011; 33: 686-90.

5. Liu B, Kuang A, Huang R, Zhao Z, Zeng Y, Wang J, Tian R. Influence of vitamin $\mathrm{C}$ on salivary absorbed dose of 131I in thyroid cancer patients: a prospective, randomized, single-blind, controlled trial. J Nucl Med. 2010; 51: 618-23.

6. Jentzen W, Balschuweit D, Schmitz J, Freudenberg L, Eising E, Hilbel T, et al. The influence of saliva flow stimulation on the absorbed radiation dose to the salivary glands during radioiodine therapy of thyroid cancer using (124)I PET(/CT) imaging. Eur J Nucl Med Mol Imaging. 2010; 37: 2298-306.

7. Aframian DJ, Helcer M, Livni D, Markitziu A. Pilocarpine for the treatment of salivary glands' impairment caused by radioiodine therapy for thyroid cancer. Oral Dis. 2006; 12: 297-300.

8. Silberstein EB. Reducing the incidence of 131I-induced sialadenitis: the role of pilocarpine. J Nucl Med. 2008; 49: 546-9.

9. Grewal RK, Larson SM, Pentlow CE, Pentlow KS, Gonen M, Qualey R, et al. Salivary gland side effects commonly develop several weeks after initial radioactive iodine ablation. J Nucl Med. 2009; 50: 1605-10.

10. Amenábar J M, Pawlowski J, Hilgert J B, Hugo FN, Bandeira $\mathrm{D}$, Lhüller F, et al. Anxiety and salivary cortisol levels in patients with burning mouth syndrome: case-control study. Oral Surg Oral Med Oral Pathol Oral Radiol Endod. 2008; 105: 460-5.

11. Van den Berg I, Pijpe J, Vissink A. Salivary gland parameters and clinical data related to the underlying disorder in patients with persisting xerostomia. Eur J Oral Sci. 2007; 115: 97-102.

12. Rosário PWS, Maia FFR, Barroso A, Padrão EL, Rezende L, Purish S. Sialoadenitis following ablative therapy with high doses of radioiodine for treatment of differentiated thyroid cancer. Arq Bras Endocrinol Metabol. 2004; 48: 310-4.

13. Rosário PWS, Cardoso LD, Barroso AL, Padrão EL, Rezende LL, Purish S. Safety of radioiodine therapy in patients with thyroid carcinoma younger than 21 years. Arq Bras Endocrinol Metabol. 2005; 49: 241-5.

14. Caglar M, Tungel M, Alpar R. Scintigraphic evaluation of salivary gland dysfunction in patients with thyroid cancer after radioiodine treatment. Clin Nucl Med. 2002; 27: 767-71.

15. Van Nostrand D, Atkins F, Bandaru VV, Chennupati SP, Moreau S, Burman K, et al. Salivary gland protection with sialogogues: A case study. Thyroid. 2009; 19: 1005-8.

16. Bomeli SR, Desai SC, Johnson JT, Walvekar RR. Management of salivary flow in head and neck cancer patients - a systematic review. Oral Oncol. 2008; 44: 1000-8. 